\title{
Ultrastructural localization of adhalin, $\alpha$-dystroglycan and merosin in normal and dystrophic muscle
}

\author{
M. J. Cullen, J. Walsh, S. L. Roberds* and K. P. Campbell* \\ Newcastle University School of Neuroscience, Muscular Dystrophy Research Laboratories, Newcastle General Hospital, \\ Newcastle-upon-Tyne, UK and *Howard Hughes Medical Institute and, Department of Physiology and Biophysics, University of \\ Iowa College of Medicine, Iowa City, Iowa, USA
}

M. J. Cullen, J. Walsh, S. L. Roberds and K. P. Campbell (1996) Neuropathology and Applied Neurobiology 22, 30-37 Ultrastructural localization of adhalin, $\alpha$-dystroglycan and merosin in normal and dystrophic muscle

Adhalin and $\alpha$-dystroglycan are two components of a complex of proteins that, in conjunction with dystrophin, provide a link between the subsarcolemmal cytoskeleton and the basal lamina of the extracellular matrix of skeletal muscle. In the absence of dystrophin, in Duchenne muscular dystrophy (DMD) and the $m d x$ mouse, levels of adhalin, $\alpha$-dystroglycan and other components of the complex, are severely reduced, and it has been speculated that this might be an important factor in precipitating myofibre necrosis. However, there is, as yet, little information on how these proteins interact structurally or functionally. From biochemical data it might be predicted that adhalin and $\alpha$-dystroglycan are positioned more peripherally in the muscle cell than dystrophin and more proximal than merosin. Using single and double immunogold labelling we here show that adhalin is localized to the plasma membrane with the majority of the gold probe particles situated on the membrane's outer face, while $\alpha$-dystroglycan labelling is seen on material which projects from the outer face and which, in places, forms strands that stretch to the basal lamina. When double labelling of laminin and $\alpha$-dystroglycan is carried out, laminin is localized to the proximal face of the basal lamina, facing the $\alpha$-dystroglycan. In DMD the labelling of adhalin and $\alpha$-dystroglycan is severely reduced quantitatively (although the vestige that remains is positioned normally) but merosin is expressed normally, showing that its incorporation is independent of that of dystrophin and its associated proteins.

Keywords: adhalin, $\alpha$-dystroglycan, merosin, dystrophin, Duchenne dystrophy

\section{Introduction}

Dystrophin, the protein absent in Duchenne muscular dystrophy (DMD), is bound to a large oligomeric complex of proteins localized to the plasma membrane $[4,7,23]$. The complex consists of a cytoplasmic protein triplet of $59 \mathrm{kDa}$ (59DAP or syntrophin), a transmembrane protein of $25 \mathrm{kDa}$ (25DAP), four transmembrane glycoproteins (35DAG, adhalin or 50DAG, and a $43 \mathrm{kDa}$ doublet [ $\beta$-dystroglycan and a novel $43 \mathrm{kDa}$ glycoprotein,

Correspondence: Dr M.J. Cullen, Newcastle University School of Neuroscience, Muscular Dystrophy Research Laboratories. Newcastle General Hospital, Westgate Road. Newcastle-upon-Tyne NE4 6BE. UK.

(C) 1996 Blackwell Science Ltd
43DAG, also known asA3b]) and an extracellular glycoprotein of $156 \mathrm{kDa}$ (156DAG or $\alpha$-dystroglycan). Alpha and $\beta$-dystroglycan are post-translationally spliced products of a single gene, so named because of their interaction with dystrophin and the heavily glycosylated nature of $\alpha$-dystroglycan [11]. In contrast to the dystroglycans which are wot specific to muscle, adhalin is expressed only in skeletal, cardiac and smooth muscle. Alpha-dystroglycan binds to merosin (M-laminin or laminin 2) in the basal lamina $[9,11]$ which is a secretion product of the myofibres and part of the extracellular matrix.

While DMD is caused by an absence of dystrophin. other disorders result from abnormalities of the 
dystrophin associated proteins. Thus, limb girdle muscular dystrophy type $2 \mathrm{C}$ is associated with adhalin deficiency and is linked with an unidentified gene on chromosome 13 [3] while limb girdle dystrophy type 2D is associated with mutations in the adhalin gene on chromosome 17 [17]. Adhalin deficiency has also been observed in the hereditary myopathy and cardiomyopathy in the hamster [16]. In Fukuyama-type congenital muscular dystrophy, reduced labelling of $\beta$-dystroglycan is seen in some fibres [13]. A proportion of cases of classical congenital muscular dystrophy (CMD) and dy/dy mouse dystrophy have recently been associated with a deficit in merosin expression [2, 19], and linkage analysis has placed the CMD locus near the merosin gene [10].

It follows from the above observations that a range of disorders that are genetically distinct but related (in that they all cause muscle necrosis) is produced by changes in components that physically span a very small part of the muscle cell, and it is therefore highly important that the morphological relationship of the different components is clarified. The resolution of immunofluorescence techniques is inadequate for this, so ultrastructural methods have been employed. Immunogold techniques have been applied in several laboratories to the localization of dystrophin $[5,20,22]$ and, more recently, to $\beta$-dystroglycan [6]. In the present study we have extended this approach to include the ultrastructural localization of adhalin, $\alpha$-dystroglycan and merosin. We have also carried out double immunogold labelling and shown that the relative disposition of the different components is largely in accord with models based on biochemical studies.

\section{Materials and methods}

\section{Antibodies}

Monoclonal

(1) Anti-adhalin antibody IVD $3_{1}$ [12]. (2) Anti- $\alpha$ dystroglycan antibody IIH6 [11]. (3) Anti-merosin (Chemicon, MAB 1922).

\section{Polyclonal}

(1) Affinity purified rabbit polyclonal antibody (P1461) raised against the last $17 \mathrm{C}$-terminus amino acids of dystrophin [5]. (donated by Dr Henry Klamut). (2) Affinity purified rabbit polyclonal to laminin (Sigma, L-9393).

\section{Muscle}

The muscle samples examined were obtained by open biopsy from the vastus lateralis muscle from patients at the Regional Neurosciences Centre, Newcastle General Hospital. Biopsy samples from six patients with DMD and 10 non-Xp21 dystrophy cases were studied. The ages of the six boys with DMD were 2, 3, 4 (two), 6 and 8 years. The non-Xp21 dystrophy cases comprised one McArdle's disease, one motor neuron disease, one polymyositis and seven cases of undiagnosed muscle weakness in which the biopsy was histologically and ultrastructurally normal.

\section{Immunolabelling}

Small pieces of vastus lateralis muscle were clamped under slight tension in modified hair grips and fixed for $1 \mathrm{~h}$ in $2 \%$ paraformaldehyde with $0.001 \%$ glutaraldehyde in phosphate buffered saline of composition $0.02 \mathrm{M}$ $\mathrm{NaH}_{2} \mathrm{PO}_{4} 2 \mathrm{H}_{2} \mathrm{O}, 0.08 \mathrm{M} \mathrm{Na}_{2} \mathrm{HPO}_{4}, 0.15 \mathrm{M} \mathrm{NaCl}$ at $\mathrm{pH}$ 7.4. After washing in buffer the muscle was cut into blocks $\approx 1.0 \times 0.5 \times 0.5 \mathrm{~mm}$ and transferred to $2.3 \mathrm{M}$ sucrose (as cryoprotectant) for at least $2 \mathrm{~h}$. The blocks were then mounted on aluminium stubs and plunge frozen in liquid nitrogen. Sections were cut at $1 \mu \mathrm{m}$ for immunofluorescence and at $80 \mathrm{~nm}$ for $\mathrm{EM}$ immunogold labelling using a Reichert Ultracut $\mathrm{E}$ microtome fitted with an FC4D cryomicrotomy attachment (knife temperature $-110^{\circ} \mathrm{C}$, block temperature $-90^{\circ} \mathrm{C}$ ). The $1 \mu \mathrm{m}$ sections were mounted on gelatinized slides, rinsed, blocked with $50 \mathrm{~mm}$ ammonium chloride and rinsed again, after which the primary antibody was applied for $1 \mathrm{~h}$ at room temperature. After thorough washing in buffer the labelling was visualized with rhodamineconjugated rabbit anti-mouse IgG (1:100; DAKO R270). After washing, the sections were mounted in Uvinert before examination using a fluorescence-optics microscope. The $80 \mathrm{~nm}$ sections were collected on to formvar/ carbon-coated 200-mesh copper grids and placed on to drops of chilled $2 \%$ gelatin to stabilize. Three consecutive blocking steps were applied using ammonium chloride, $50 \mathrm{~mm}$ for $15 \mathrm{~min}$, glycine, $0.02 \mathrm{M}$ for $15 \mathrm{~min}$ and $10 \%$ normal goat serum for $30 \mathrm{~min}$. The grids were then floated on drops of primary antibody for $1 \mathrm{~h}$. After washing in buffer the grids were incubated with the gold-conjugated secondary antibody for $1 \mathrm{~h}$ at room temperature. After further washing the sections were stabilized in $2 \%$ methyl cellulose containing $0.4 \%$ uranyl

(C) 1996 Blackwell Science Ltd, Neuropathology and Applied Neurobiology, 22, 30-37 
acetate and allowed to air dry. The sections were examined in a JEOL 1200EX electron microscope.

\section{Controls}

When labelling with a monoclonal antibody, control sections were incubated, in parallel, with diluted nonimmune mouse serum followed by the appropriate goat anti-mouse gold conjugate. Other control sections were incubated with the buffer alone followed by the gold conjugate. The same controls were carried out when labelling with the polyclonal antibodies except that non-immune rabbit serum was used.

\section{Double labelling}

To carry out double labelling, the sections were first labelled by the usual procedure with one of the antibodies (monoclonal or polyclonal) and the appropriate goldconjugate and, after washing, were then labelled with the second antibody (raised in a different species to the first antibody) and a gold-conjugate of a different size to that used for the first antibody. Due to the unavailability of a polyclonal antibody to merosin at the time, double labelling of $\alpha$-dystroglycan and the basal lamina was carried out using a polyclonal antibody to laminin. (The monoclonal antibody recognizes the $\alpha 2$ chain of laminin while the polyclonal recognizes more than one chain but not the $\alpha 2$. Thus while the epitopes of the two antibodies are different they will label the same molecules.) As a control for the double labelling, the position and density of labelling was compared with that for single labelling with each antibody. In order to control for possible crossreactivity or steric hindrance, the labelling for the two antibodies was also carried out in the reversed sequence.

\section{Results}

\section{Control muscle}

Adhalin

Immunofluorescence labelling of the control muscle showed strong labelling with IVD $3_{1}$ at the periphery of the fibres (Figure 1a).

At the ultrastructural level, immunogold labelling was seen mostly close to the plasma membrane and almost entirely on the outer face of it (Figure 2). Faint strands of material could often be seen stretched between the basal lamina and plasma membrane, and the adhalin labelling was often seen near the base of these strands (Figure 2).

\section{Alpha-dystroglycan}

Immunofluorescence labelling of the control muscle showed very intense labelling with $11 \mathrm{H} 6$ at the periphery of the fibres (Figure 1b). Where the basal lamina had pulled away from the underlying fibre, probably during sectioning, a low intensity fluorescence was associated with the lamina. Ultrastructurally, immunogold labelling was found on the outer side of the plasma membrane, usually at a site where material that was denser than the background could be seen projecting from the outer face (Figure 3). There was frequent multiple labelling by the gold at each site, which we attribute to $11 \mathrm{H} 6$ being an IgM class molecule and therefore, as a pentamer, possessing more antigenic sites for binding by the goldconjugated secondary antibody.

\section{Merosin}

By immunofluorescence, anti-merosin clearly defined the periphery of each muscle fibre (Figure 4). At the ultrastructural level, immunogold labelling was confined to the basal lamina, and within the basal lamina itself labelling was mainly located at the side facing the plasma membrane (Figure 5). In places, strands of material could be detected connecting the basal lamina and plasma membrane and these were often associated with immunogold labelling close to the basal lamina end (Figure 5). Immunogold labelling of laminin was indistinguishable from that of merosin.

\section{Alpha-dystroglycan and dystrophin}

When double labelling of $\alpha$-dystroglycan and dystrophin was carried out, a clear topographical segregation of the two labelling sites could be distinguished. In Figure 6. for example, the $15 \mathrm{~nm}$ immunogold labelling $\alpha$-dystroglycan is all positioned external to the plasma membrane while the $10 \mathrm{~nm}$ gold labelling dystrophin is mostly either on the membrane or immediately internal to it. In places an approximate correspondence can be seen between the labelling sites on either side of the membrane.

\section{Alpha-dystroglycan and laminin}

Double labelling was carried out using $5 \mathrm{~nm}$ immunogold to localize the $\alpha$-dystroglycan and $10 \mathrm{~nm}$ gold to label laminin (Figure 7). The $\alpha$-dystroglycan was strongly labelled and in places appeared to be pulled out in strands that cross the gap between the plasma membrane and the basal lamina. 

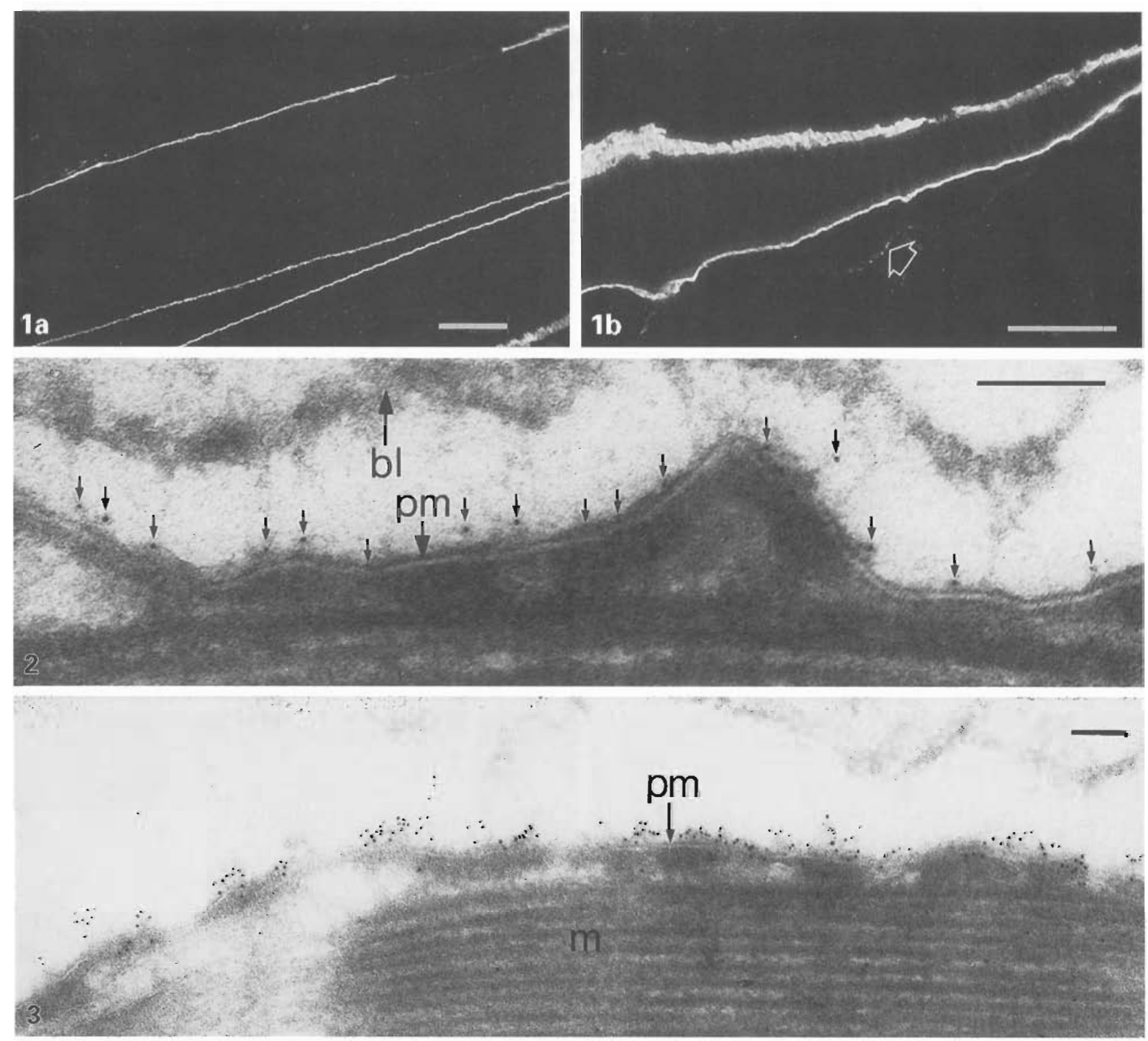

Figure 1. a, An example of IVD3, labelling of control muscle using immunofluorescence. Adhalin is confined to the periphery of the fibres. b, IIH6 labelling of control muscle by immunofluorescence. $\alpha$-Dystroglycan is largely confined to the periphery of the fibre but there is also weak labelling where the basal lamina has lifted off the the fibre (arrow). Bar $=20 \mu \mathrm{m}$.

Figure 2. An example of adhalin labelling of control muscle using $5 \mathrm{~nm}$ immunogold. The labelling is close to and immediately peripheral to the plasma membrane (pm) and is often associated with the base of strands of material that stretch between the membrane and the basal lamina (bl). Bar $=100 \mathrm{~nm}$.

Figure 3. An example of $\alpha$-dystroglycan labelling of control muscle using $5 \mathrm{~nm}$ immunogold. The labelling sites are on the outer side of the plasma membrane (pm) where material projects from the face of the membrane. There is multiple labelling of the sites because IIH6 is an IgM class antibody. $\mathrm{m}=$ myotibril. Bar $=100 \mathrm{~nm}$.

\section{DMD muscle}

Immunofluorescence labelling of adhalin in DMD muscle showed a severe reduction in intensity when compared with control muscle (Figure 8a). The label that remained encompassed the whole of the fibres with only minor variations in intensity. At the ultrastructural level immunogold particles were found in the same position as in control muscle, close to and usually external to the plasma membrane, but at a far lower density than in controls. Immunofluorescence labelling of 

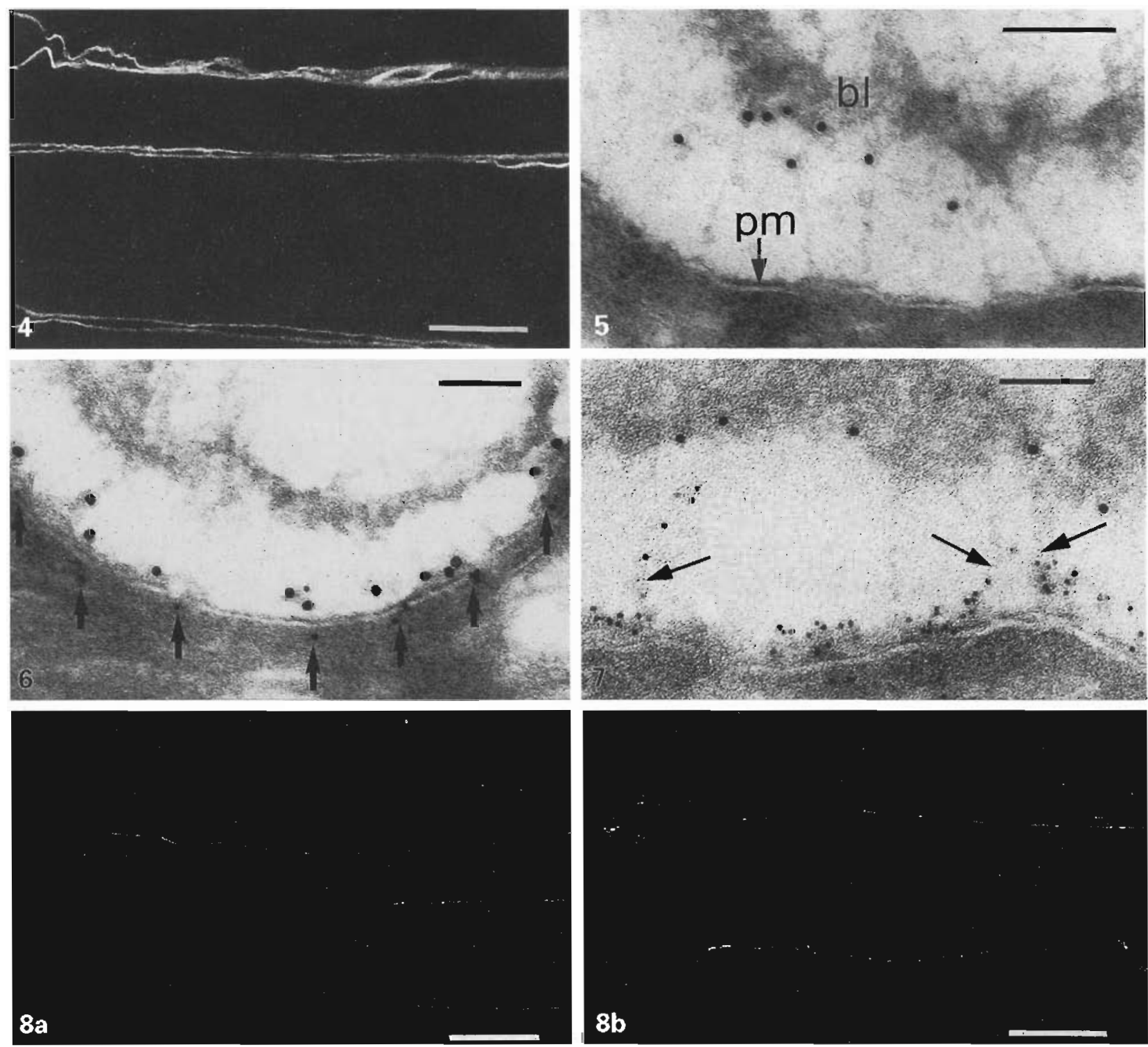

Figure 4. Immunofluorescence labelling of merosin in control muscle. The edges of the fibres are clearly defined. Bar $=20 \mu \mathrm{m}$.

Figure 5. Immunogold labelling of merosin showing a concentration of label on the side of the basal lamina (bl) facing the plasma membrane (pm). Faint strands connect the basal lamina and plasma membrane, often with immunogold at the basal lamina end. $10 \mathrm{~nm}$ gold. Bar $=100 \mathrm{~nm}$.

Figure 6. Double immunogold labelling of dystrophin (10 nm gold) and $\alpha$-dystroglycan ( $15 \mathrm{~nm}$ gold) in control muscle. The $\alpha$-dystroglycan label lies outside the plasma membrane while the dystrophin label is on the membrane or internal to it (arrows). Bar $=100 \mathrm{~nm}$.

Figure 7. Double immunogold labelling of $\alpha$-dystroglycan ( $5 \mathrm{~nm}$ gold) and laminin ( $10 \mathrm{~nm}$ gold) in control muscle. The $\alpha$-dystroglycan label is largely on the outside of the plasma membrane and in places is seen on strands (arrows) stretching towards the basal lamina. Bar $=100 \mathrm{~nm}$.

Figure 8. a, Immunofluorescence labelling of adhalin in a DMD patient. The labelling is fairly uniform but severely reduced when compared with controls. $b$, Immunofluorescence labelling of $\alpha$-dystroglycan in DMD. The labelling is considerably less intense than in control muscle and less uniform than that of adhalin. Bars $=20 \mu \mathrm{m}$. 
$\alpha$-dystroglycan, as with adhalin, showed a severe reduction in intensity when compared with controls (Figure 8b). The remaining labelling, however, was less uniform and more patchy compared with that of adhalin. At the ultrastructural level, immunogold labelling was found in the same position as in controls but at a notably lower density. The merosin labelling of the basal laminae of myofibres in DMD appeared as strong as that in controls by both immunofluorescence and immunogold labelling.

\section{Discussion}

The aim of this study was to investigate the normal location of adhalin and $\alpha$-dystroglycan and to examine to what extent this was altered in dystrophic muscle in which there is an absence of dystrophin. Based on a large amount of biochemical evidence, several schematic models have been proposed for the structure of the dystrophin-glycoprotein complex at the plasma membrane $[1,8,14,18]$. There are a number of specific differences between these models, but certain general features are common to each one. Thus, in each model, $\alpha$-dystroglycan is considered to be positioned on the extracellular side of the plasma membrane where it binds to merosin located in the basal lamina, and, adhalin, while classified as an integral membrane protein, is considered to have a large extracellular component. (There is biochemical evidence that the adhalin antibody used in this study, IVD3, recognizes an epitope in this extracellular region [unpublished data].) The results described in this paper give strong morphological support to these general models of the dystrophinglycoprotein complex. Immunogold labelling of $\alpha$ dystroglycan shows very clear localization on the extracellular side of the plasma membrane (Figure 3). Immunofluorescence labelling of adhalin appears very similar to that of $\alpha$-dystroglycan and the immunogold labelling clearly defines an epitope in the extracellular domain of the molecule (Figure 2). Where double labelling of $\alpha$-dystroglycan and dystrophin was carried out, the gold particles labelling $\alpha$-dystroglycan were frequently in the vicinity of gold particles labelling dystrophin with the $\alpha$-dystroglycan gold nearly invariably external to the plasma membrane and the dystrophin gold internal to it or overlying it. The relative positions of $\alpha$-dystroglycan and dystrophin were therefore in accord with the models depicted in the literature (Figure 9).

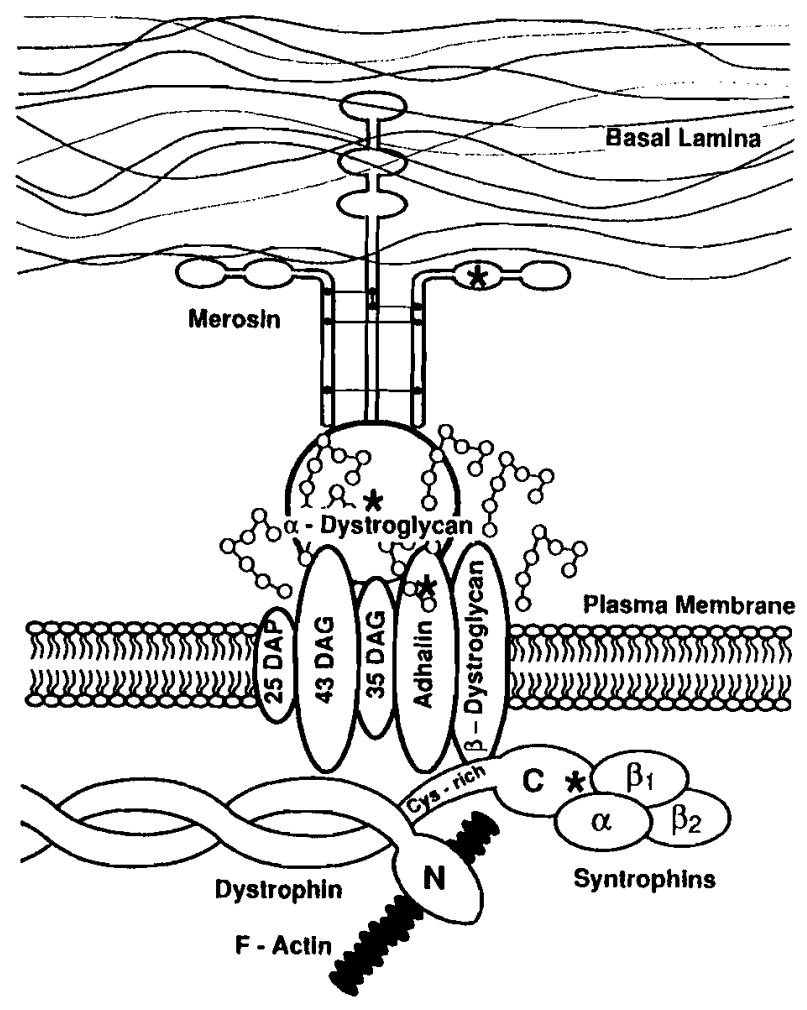

Figure 9. Schematic model of the dystrophin-glycoprotein complex. Asterisks mark the components labelled in this study.

Immunofluorescence labelling of merosin and laminin (not shown) clearly defined the periphery of the muscle fibres. The immunogold labelling, with localization mostly on the side of the basal lamina facing the plasma membrane, is in accord with the schematic models (Figure 9). The immunogold particles were often situated at the basal lamina ends of pale strands of material that linked the plasma membrane and the basal lamina (Figure 5). These strands could represent part of protein molecules or carbohydrate moieties involved in connecting the two structures. Double labelling experiments, in which both laminin and $\alpha$-dystroglycan were labelled, show $\alpha$-dystroglycan label clustered at the base of the strands (Figure 7) which suggests that they may represent the carbohydrate component extensions to $\alpha$-dystroglycan. Moreover adhalin was frequently localized to the base of the strands (Figure 2) which further suggests that they mark the position of the glycoprotein complex.

The dimensions of the gap (sometimes termed the lamina lucida) between the plasma membrane and the basal lamina, is very variable and partly dependent on 
the method of preparation of the muscle. We consider that in the present preparations it is exaggerated and that the two layers in vivo are almost certainly more closely adjacent, and the two epitopes labelled, for example in Figure 7, are more closely positioned than appears in the micrographs. We do not, however, consider that the strands of material connecting the plasma membrane and basal lamina are artefactual as these have been described in muscle prepared in different ways [21].

\section{Dystrophic muscle}

Immunofluorescence (Figure 8) and immunogold labelling of both adhalin and $\alpha$-dystroglycan was considerably reduced in the DMD patients compared with controls. The immunogold labelling of both seemed more reduced than that seen with $\beta$-dystroglycan labelling of DMD cases [6]. although this was less obvious from the immunofluorescence staining. The patchiness of the immunofluorescence staining of the $\alpha$-dystroglycan compared with the adhalin was similar to that seen with $\beta$-dystroglycan [6]. This suggests that the absence of dystrophin may affect the incorporation of $\alpha$ - and $\beta$-dystroglycan at the plasma membrane differently to adhalin. This might be expected if the binding of dystrophin in the DGC is via $\beta$-dystroglycan and this, in turn, binds $\alpha$-dystroglycan [18] whereas adhalin may be more removed from the dystrophin binding site. It does not, however, explain why adhalin and other DAGs are also so greatly reduced in DMD [7, 15]. Finally, and in relation to this, the loss of which particular component of the DGC initiates cell necrosis in DMD remains to be determined.

\section{Acknowledgements}

M.J.C. and J.W. acknowledge financial support from the Muscular Dystrophy Group of Great Britain. This work was also supported in part by the Muscular Dystrophy Association (USA). S.L.R. is the Paul Cohen Neuromuscular Disease Research Fellow of the Muscular Dystrophy Association, and K.P.C. is an investigator of the Howard Hughes Medical Institute.

\section{References}

1 Ahn AH, Kunkel LM. The structural and functional diversity of dystrophin. Nature Genet 1993; 3: 283-91
2 Arahata K, Hayashi KY, Koga R et al. Lamin in animal models for muscular dystrophy: defect of laminin $M$ in skeletal and cardiac muscles and peripheral nerve of the homozygous dystrophic dy/dy mice. Proc Jap Acad 1993 69B: 259-64

3 Azibi K, Bachner L, Beckmann IS et al. Severe childhood autosomal recessive muscular dystrophy with the deficiency of the $50 \mathrm{kDa}$ dystrophin-associated glycoprotein maps to chromosome 13q12. Hum Mol Genet 1993; 2: 1423-8

4 Campbell KP, Kahl SD. Association of dystrophin and an integral membrane glycoprotein. Nature 1989; 338: 259-62

5 Cullen MJ, Walsh J, Nicholson LVB et al. Immunogold labelling of dystrophin in human muscle, using an antibody to the last 17 amino acids of the C-terminus. Neuromusc Disord 1991; 1: 113-19

6 Cullen MJ, Walsh J. Nicholson LVB. Immunogold localization of the $43 \mathrm{kDa}$ dystroglycan at the plasma membrane in control and dystrophic human muscle. Acta Neuropathol 1994; 87: 349-54

7 Ervasti JM, Ohlendieck K, Kahl SD, Gaver MG, Campbell KP. Deficiency of a glycoprotein component of the dystrophin complex in dystrophic muscle. Nature 1990: 345: 315-19

8 Ervasti JM, Campbell KP. Membrane organization of the dystrophin-glycoprotein complex. Cell 1991; 66: 1121-31

9 Ervasti JM, Campbell KP. A role for the dystrophinglycoprotein complex as a transmembrane linker between laminin and actin. J Cell Biol 1993; 122: 809-23

10 Hillaire D, Leclerc A, Faure S et al. Localization of merosinnegative congenital muscular dystrophy to chromosome 6 q2 by homozygosity mapping. Hum Mol Genet 1994; 3: 1657-61

11 Ibraghimov-Beskrovnaya O, Ervasti JM, Leveille CJ, Slaughter CA, Sernett SW, Campbell KP. Primary structure of dystrophin-associated glycoproteins linking dystrophin to the extracellular matrix. Nature 1992; 355: 696-702

12 Jorgensen AO, Arnold W. Shen AC-Y, Yuan S, Gaver M, Campbell KP. Identification of novel proteins unique to either transverse tubules (TS28) or the sarcolemma (SL50) in rabbit skeletal muscle. J Cell Biol 1990; 110: 1173-85

13 Matsumura K, Nonaka I, Campbell KP. Abnormal expression of dystrophin-associated proteins in Fukuyama-type congenital muscular dystrophy. Lancet 1993b; 341: 521-2

14 Matsumura K, Campbell KP. Dystrophin-glycoprotein complex: its role in the molecular pathogenesis of muscular dystrophies. Muscle Nerve 1994; 17: 2-15

15 Mizuno Y, Yoshida M, Nonaka I, Hirai S, Ozawa E. Expression of utrophin (dystrophin-related protein) and dystrophin-associated glycoproteins in muscles from patients with Duchenne muscular dystrophy. Muscle Nerve 1994; 17: 206-16 
16 Roberds SL, Ervasti JM, Anderson RD et al. Disruption of the dystrophin-glycoprotein complex in the cardiomyopathic hamster. J Biol Chem 1993; 268: 11496-9

17 Roberds SL, Leturcq F, Allamand V et al. Missense mutations in the adhalin gene linked to autosomal recessive muscular dystrophy. Cell 1994; 78: 625-33

18 Suzuki A. Yoshida M, Hayashi K, Mizuno Y, Hagiwara Y, Ozawa E. Molecular organization of the glycoproteincomplex-binding site of dystrophin. Three dystrophinassociated proteins bind directly to the carboxy-terminal portion of dystrophin. Europ $J$ Biochem 1994: 220: 283-92

19 Tomé FMS, Evangelista T, Leclerc A et al. Congenital muscular dystrophy with merosin deficiency. Comp Rend Acad Sci Paris 1994: 317: 351-7
20 Wakayama Y. Shibuya S. Gold-labelled dystrophin molecule in muscle plasmalemma of $\mathrm{mdx}$ control mice as seen by electron microscopy of deep etching replica. Acta Neuropathol 1991; 82: 178-84

21 Watkins SC, Cullen MJ. Histochemical fibre typing and ultrastructure of the small fibres in Duchenne muscular dystrophy. Neuropathol Appl Neurobiol 1985; 11: 447-60

22 Watkins SC, Hoffman EP, Slayter HS, Kunkel LM. Immunoelectron microscopic localization of dystrophin in myofibres. Nature 1988; 333: 863-6

23 Yoshida M, Ozawa E. Glycoprotein complex anchoring dystrophin to sarcolemma. J Biochem 1990; 108: 748-52

Received 11 April 1995

Accepted after revision 20 August 1995 